\title{
Management of OCDMA auto-correlation function distorted by dispersion effects
}

\author{
Md Shakil Ahmed, Mohamed Abuhelala, Ivan Glesk, Senior Member, IEEE \\ University of Strathclyde, Glasgow, UK \\ Tel: +4401415482529,e-mail: ivan.glesk@strath.ac.uk
}

\begin{abstract}
Dispersion has very strong detrimental effects on high data rate incoherent transmission systems. Situation gets much worse when data bits become only a few picoseconds long. In this paper we investigate how the dispersion can affect an optical auto-correlation function in optical code division multiple access (OCDMA) systems when data are carried by two-dimensional wavelength-hopping time-spreading codes based on multi-wavelength picosecond pulses. We show how chromatic dispersion and changes of optical fiber temperature can distort the OCDMA auto-correlation. We also demonstrate a simple method for efficient mitigation of these effects.

Keywords: optical communication, chromatic dispersion, temperature induced dispersion, semiconductor optical amplifier, optical pulse/holding beam, temporal skewing.
\end{abstract}

\section{INTRODUCTION}

Fiber optic communication systems use different multiplexing techniques such as optical time division multiplexing (OTDM), wavelength division multiplexing (WDM) and optical code division multiplexing (OCDM) to carry data. In OCDM, each communication channel is distinguished by a unique optical code rather than a wavelength or time-slot. For increasing the number of simultaneous users and /or enhancing data privacy, especially in a network transport layer, OCDM is a promising option [1]. OCDMA systems can be design to use codes based on multi-wavelength picosecond optical pulses as the data carriers. One example would be a coding based on Two-Dimensional Wavelength-Hopping Time-Spreading (2D-WH/TS) family of codes. Here a selected number of multi-wavelength picosecond pulses is arranged in both, wavelength and time domain to form a family of codes for use by a varied number of network users. At a receiver side the incoming data are recovered for each user by a decoding process which produces a so called OCDMA auto-correlation function (see Fig. 1(a)). During data transmission is very important to preserve its undistorted shape in order to maintain the highest performance of OCDMA systems [2].

Dispersion effects on optical fiber are well known and may not be neglected when a pulse width of data carriers becomes narrow which is the case of incoherent high rate data transmission [3]. As already mentioned, in 2D-WH/TS OCDMA systems, the multi-wavelength picosecond pulses are distributed within a data bit-width to form an OCDMA code. Due to chromatic dispersion (CD) or temperature-induced dispersion (TD) these short multi-wavelength pulses propagating in the optical fiber will be affected by a so called time-skewing effect. As a result, at the receiver end, the recovered OCDMA auto-correlation function becomes distorted. It changes its shape, notably its width (see Fig. 1). This is illustrated in Fig. 1(b). This can drastically influence the OCDMA system performance in terms of BER and the total number of simultaneous users [4], [5]. It is therefore necessary to control and manage CD and TD.

Normally a dispersion compensating fibre (DCF) is used to control CD. This approach would be quite time consuming if $\mathrm{CD}$ compensation with the picosecond accuracy is required [3]. Therefore there is a need for a more practical dispersion management to prevent the OCDMA auto-correlation function distortion by CD or TD effects.

In this paper, we will first demonstrate how $\mathrm{CD}$ or TD can affect the OCDMA auto-correlation function as a result of data propagation in optical fiber of a varying length and / or under temperature changes. Then we will show a simple approach how these effects can be mitigated.

\section{EFFECTS OF CD AND TD ON OCDMA AUTO-CORRELATION FUNCTION}

In order to perform our investigations an OCDMA testbed based on 2D-WH/TS coding scheme using multiwavelengths picosecond code carriers was constructed (see Fig. 2a) using a $19.5 \mathrm{~km}$ long spool of SMF-28 optical fiber as the transmission link. An erbium doped fiber mode-locked laser system was used to generate an optical supercontinuum which was spectrally sliced by a fiber Bragg grating (FBG) OCDMA encoder to produce a $2 \mathrm{D}-\mathrm{WH} / \mathrm{TS}$ OCDMA code. In our investigations we used the USER-1 code $\left(1-\lambda_{2}, 21-\lambda_{4}, 24-\lambda_{1}, 39-\lambda_{3}\right)$ where $\lambda_{1}=1551.72 \mathrm{~nm}, \lambda_{2}=1550.92 \mathrm{~nm}, \lambda_{3}=1552.52 \mathrm{~nm}$, and $\lambda_{4}=1550.12 \mathrm{~nm}$. Here, the leading number represents the chip order.

a) In order to investigate how the 2D-WH/TS OCDMA auto-correlation function can be affected by an improper $\mathrm{CD}$ compensation, the transmission link was first fully $\mathrm{CD}$ compensated with a sub-picosecond accuracy using DCF [6]. Then its length was 'slightly' varied from its full CD compensation during our measurements. 
b) To investigate TD influence on the OCDMA auto-correlation function the $19.5 \mathrm{~km}$ long spool of SMF-28 fiber representing the transmission link was placed into an environmental chamber and then the chamber temperature was varied. During our investigations DCF used for the link full CD compensation was kept at the constant room temperature of $25^{\circ} \mathrm{C}$ out of the chamber.

\subsection{Investigation of OCDMA Auto-correlation Distortion Due To Fibre Chromatic Dispersion}

In this investigation, we kept the $19.5 \mathrm{~km}$ fiber link at the room temperature of $25^{\circ} \mathrm{C}$ and slightly varied the transmission link's length from its full CD compensation by adding/removing $50 \mathrm{~m}$ of SMF28. At the receiving end the signal was then decoded by a matched OCDMA decoder. Figure 1(c) and 1(d) are the examples of a distorted OCDMA auto-correlation function when the transmission link was not properly CD compensated due to subtracting (which resulted in over-compensation) or adding (resulted in under-compensation) about a $50 \mathrm{~m}$ of SMF-28. For the better clarity Fig. 1(a) illustrates an undistorted OCDMA auto-correlation function which in our experiments was composed of four multi-wavelength code carriers $\lambda_{1}$ to $\lambda_{4}$. Figure 1 (b) shows its distortion due to skewing resulted from the incomplete CD compensation of the transmission link. The time-skewing among wavelength code carriers $\lambda_{1}$ to $\lambda_{4}$ is a result of group velocity changes induced by the incomplete CDC.

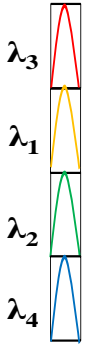

(a)

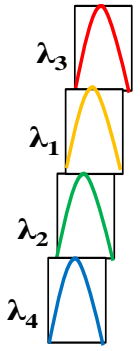

(b)

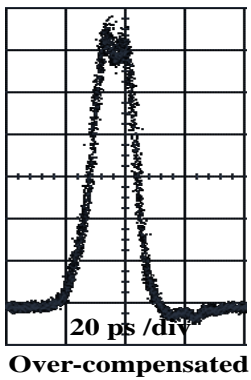

(c)

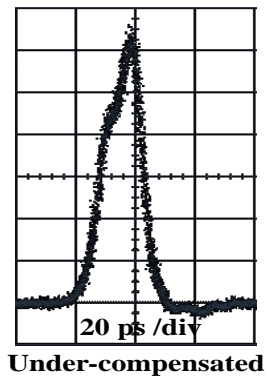

(d)

Figure 1a. Illustration of OCDMA auto-correlation function; (b) Illustration of its distorted shape by skewing due to CD mismatch; $(c)$ and $(d)$ obtained result. (Measurements were taken at a room temperature of $25^{\circ} \mathrm{C}$ ).

To overcome the influence of CD on the OCDMA auto-correlation the use of SOA for its reshaping was investigated. For this investigation we used an experimental setup shown in Fig. 2(a). First, using a back-to-back measurement the FWHM value of the OCDMA auto-correlation function was determined as 10 ps. Then we measured the FWHM of the OCDMA auto-correlation function for data transmission in a partially CD compensated testbed. The value found was $16 \mathrm{ps}$. Next, our goal was to investigate whether the OCDMA autocorrelation FWHM could be reverted back to its original value of $10 \mathrm{ps}$ seen during the back-to-back measurement. To do this, we used an optical semiconductor amplifier (SOA) and a 2 ps optical pulse as an optical holding beam (OP/HB). By controlling the optical power of OP/HB injected synchronously via an optical delay line (see ODL in Fig. 2(a)) in-line with the recovered OCDMA auto-correlation at the SOA input (point D), a FWHM value was measured at point F by an oscilloscope (OSC). Then the level of CD compensation was altered and the measurement was repeated. Band-pass filters BPF-1 and BPF-2 were used to eliminate out-ofband ASC. Figure 2(b) shows the obtained results. Here, the OCDMA Auto-correlation Width Ratio value

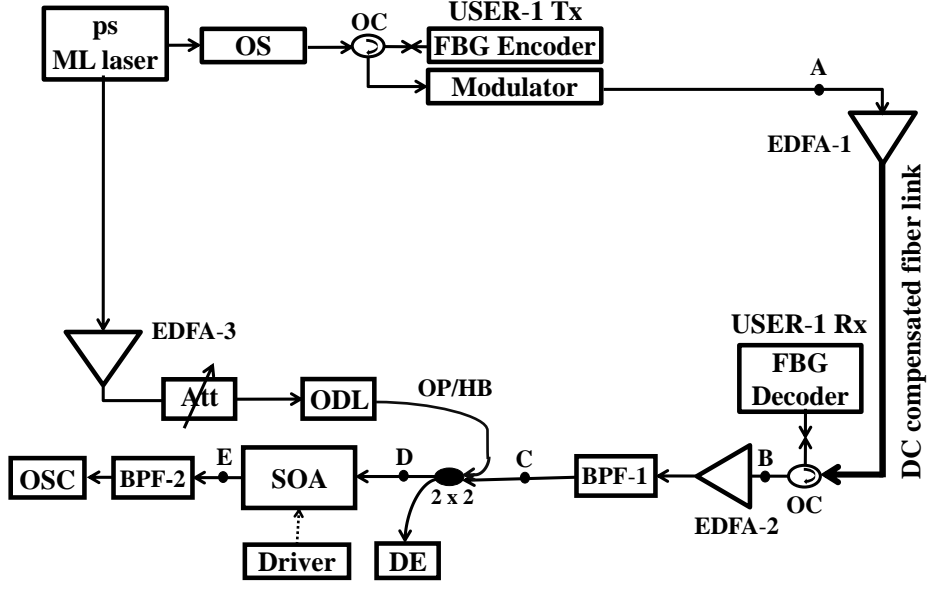

(a)

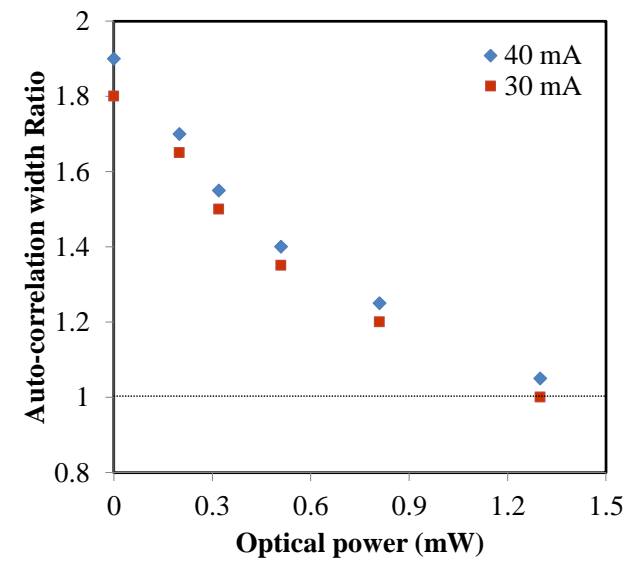

(b)

Figure 2a. Experimental setup for using SOA to control OCDMA auto-correlation distorted by chromatic dispersion; b) CD Compensation via SOA Control. OS - optical supercontinuum, OSC-oscilloscope, Attoptical attenuator, BPF - optical band pass filter, ODL - optical tuneable delay line, DE-optical power meter, $S O A$ - semiconductor optical amplifier, $O C$-optical circulator, FBG - fiber Bragg grating. 
defined as the measured auto-correlation FWHM value divided by its back-to-back value of 10 ps are plotted as a function of the OP/HB optical power for two different SOA drive currents (30 mA and $40 \mathrm{~mA})$. From Fig. 2(b) it is clear that for the drive current of $30 \mathrm{~mA}$ and OP/HB optical power of $1.3 \mathrm{~mW}$ the OCDMA auto-correlation FWHM value is reversed back to its original value of 10 ps we saw during the back-to-back measurements (i.e., the OCDMA Auto-correlation Width Ratio is equal to 1).

\subsection{OCDMA Auto-correlation Distortion Due To Transmission Link Temperature Changes}

Optical fiber links are buried at a depth of approximately three feet below the ground. Seasonal temperature variations of about $20{ }^{\circ} \mathrm{C}$ can be seen at this depth [7]. The temperature induced dispersion (TD) for SMF-28 has been determined to be $-0.0015 \mathrm{ps} / \mathrm{nm} / \mathrm{km} /{ }^{\circ} \mathrm{C}$ [8]. This value seems to be negligible compared to a $\mathrm{CD}$ value of $+17 \mathrm{ps} / \mathrm{nm} / \mathrm{km}$ for SMF-28. But even this small value of TD can affect OCDMA systems based on multiwavelengths picosecond code carriers even when the fiber $\mathrm{CD}$ is fully compensated. This varying temperature induced dispersion will affect the propagating 2D-WH/TS OCDMA codes by producing the time-skewing among code carriers which will then lead to the OCDMA auto-correlation function distortion [5].

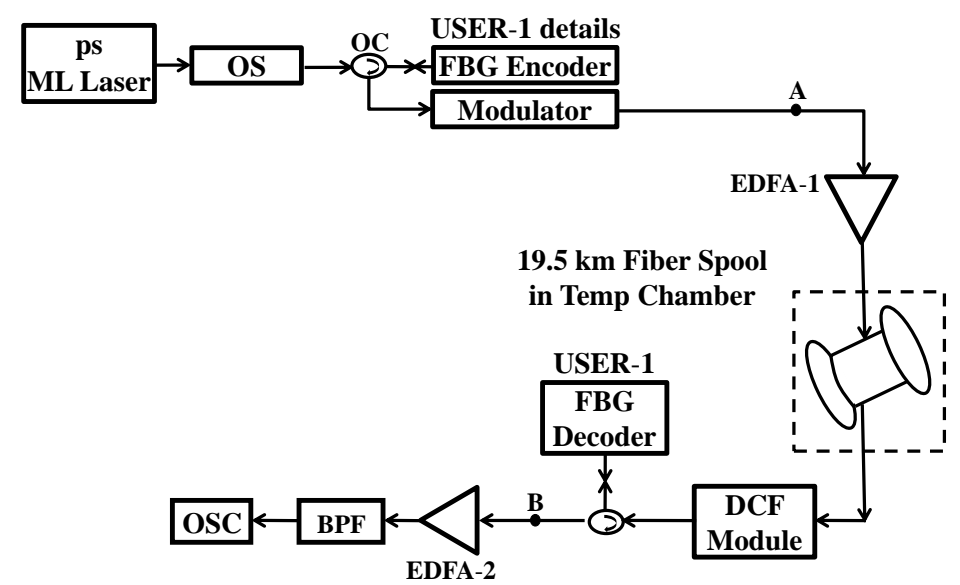

Figure 3. Experimental setup for investigating OCDMA auto-correlation distorted by temperature induced dispersion in optical fibre.

In order to investigate the sole effects of the temperature induced dispersion on OCDMA systems using 2DWH/TS codes based on multi-color picosecond pulses, we placed a $19.5 \mathrm{~km}$ fiber link (a spool of SMF-28) inside of the environmental chamber. The DCM module used to fully compensate for the link CD was kept at the room temperature outside of the chamber (see Fig. 3). The recovered OCDMA auto-correlation function on the receiver side was observed by an oscilloscope, OSC. As OSC we used a Digital Communication Analyser Agilent Infiniium DCA-J 86100C with a 64 GHz optical sampling head). The measurements are in Fig. 4(a) and Fig. 4(b) and show the received OCDMA auto-correlation functions affected by the data transmission in optical fiber kept at $5{ }^{\circ} \mathrm{C}$ and $45{ }^{\circ} \mathrm{C}$, respectively.

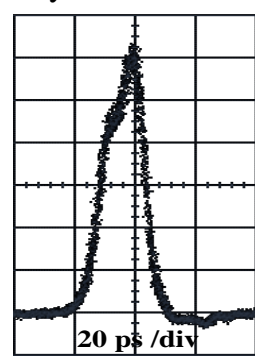

At $5^{\circ} \mathrm{C}$

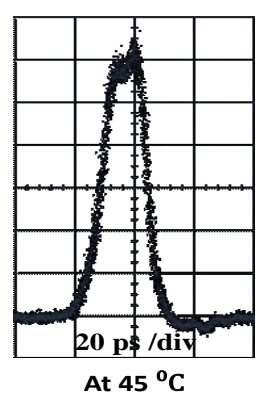

(b)

Figure 4. Temperature induced autocorrelation distortion due to different fiber temperature. (a) $5{ }^{0} \mathrm{C}$; (b) $45^{0} \mathrm{C}$.

Then we compared the results in Fig. 1 with those in Fig. 4. We found that at the fiber temperature of $45^{\circ} \mathrm{C}$ (Fig. 4(b)) the auto-correlation function's shape becomes similar to the shape we have seen in Fig. 1(c). Similarly, for the fiber temperature $5{ }^{\circ} \mathrm{C}$ (Fig. 4(b)), the auto-correlation function's shape becomes similar to the one seen in Fig. 1(d). This means, that increases in the fiber temperature make the originally fully CD compensated fiber link 'over-compensated' and decreases in the fiber temperature will make it 'under-compensated'. In this particular case, in order to make the fiber link fully dispersion compensated again at $5{ }^{0} \mathrm{C}\left(45{ }^{0} \mathrm{C}\right)$, it would require removing (adding) approximately $\sim 80 \mathrm{~m}$ of SMF-28 to its original length, respectively. 


\subsection{Measurement of Temperature Induced Skewing}

Next we decided to determine the time-skewing i.e., the temperature induced delay between the two $2 \mathrm{D}-\mathrm{WH} / \mathrm{TS}$ wavelength code carriers $\lambda_{1}=1551.72 \mathrm{~nm}$ and $\lambda_{4}=1550.12 \mathrm{~nm}$ being spectrally separated by $1.6 \mathrm{~nm}$. We used the same experimental setup as is shown in Fig. 3. These measurements were done by a Digital Communication Analyser (Agilent Infiniium DCA-J $86100 \mathrm{C}$ with a $64 \mathrm{GHz}$ optical sampling head) for a range of fiber link temperatures from $25.8{ }^{\circ} \mathrm{C}$ to $45.8{ }^{\circ} \mathrm{C}$. Data were taken with $4{ }^{\circ} \mathrm{C}$ increments, using the environmental chamber. We kept at least 2.5 hours between measurements to ensure temperature equilibrium. Our obtained results are shown in Fig. 5.

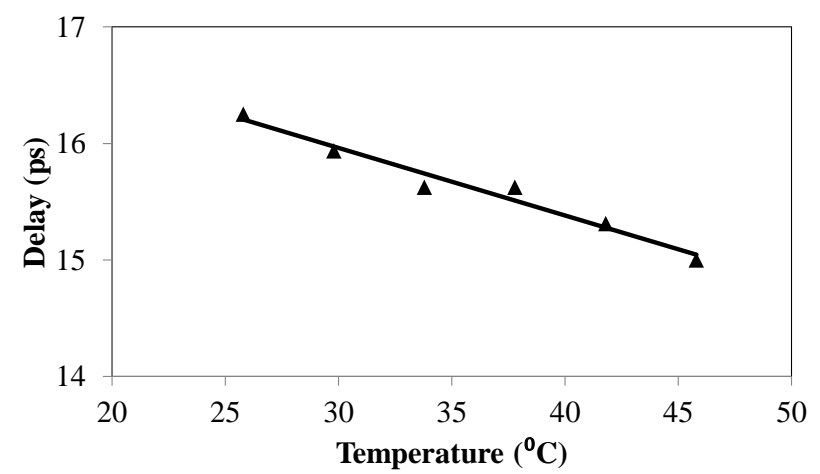

Figure 5. Time skewing between code carriers $\lambda_{1}=1551.72 \mathrm{~nm}$ and $\lambda_{4}=1550.12 \mathrm{~nm}$ spectrally separated by $1.6 \mathrm{~nm}$ as a function of different fiber temperatures.

\section{CONCLUSIONS}

In this paper we have investigated the effects of chromatic dispersion (CD) and temperature induced dispersion (TD) on the OCDMA auto-correlation function during the data transmission in SMF-28 optical fiber. Skewing between two wavelengths code carriers was measured as a function of fibe link temperature. We have shown that an SOA can be successfully used to compensate a CD affected OCDMA auto-correlation function. By investigating TD effects on OCDMA data transmission we have demonstrated that an increase /decrease of fiber temperature will over-compensate /under-compensate the transmission's link dispersion thus negatively impacting the OCDMA auto-correlation function which would lead to system performance degradations.

\section{ACKNOWLEDGEMENT}

This project has received funding from the European Union's Horizon 2020 research and innovation programme under the Marie Skłodowska-Curie grant agreement No 734331.

\section{REFERENCES}

[1] K. Fouli and M. Maier: Ocdma and optical coding: Principles, applications, and challenges [topics in optical communications], IEEE Communications Magazine, vol. 45, pp. 27-34, 2007.

[2] X. S. Yao, J. Feinberg, R. Logan, and L. Maleki: Limitations on peak pulse power, pulse width, and coding mask misalignment in a fiber-optic code-division multiple-access system, J. Lightwave Technol., vol. 11, pp. 836-846, 1993.

[3] Z. Pan, C. Yu, and A. E. Willner: Optical performance monitoring for the next generation optical communication networks, Optical Fiber Technology, vol. 16, pp. 20-45, 2010.

[4] A. Sahin and A. E. Willner: System limitations due to chromatic dispersion and receiver bandwidth for 2-D time-wavelength OCDMA systems, in Proc. Lasers and Electro-Optics Society, 2003. LEOS 2003, The 16th Annual Meeting of the IEEE. Vol. 2. IEEE, 2003.

[5] T. B. Osadola, et al.: Effect of variations in environmental temperature on 2D-WH/TS OCDMA code performance, J. Opt. Commun. Netw., vol. 5, pp. 68-73, 2013.

[6] M. S. Ahmed and I. Glesk: Recent advances in all-optical signal processing for performance enhancement of interconnects, in Proc. 18th International Conference on Transparent Optical Networks ICTON, 2016, Trento, Italy. Invited. DOI: 10.1109/ICTON.2016.7550537.

[7] A. Walter and G.S. Schaefer: Chromatic dispersion variations in ultra-long-haul transmission systems arising from seasonal soil temperature variations, in Proc. OFC 2002, pp. 332- 333, 17-22 Mar. 2002.

[8] G. Ghosh, M. Endo, and T. Iwasaki: Temperature-dependent Sellmeier coefficients and chromatic dispersions for some optical fiber glasses, J. Light. Technol., vol. 12, no. 8, pp. 1338-1342, Aug. 1994.

[9] T. Kato, Y. Koyano, and M. Nishimura: Temperature dependence of chromatic dispersion in various types of optical fiber, Opt. Let., vol. 25, no. 16, pp. 1156-1158, Aug. 2000. 